\title{
Article \\ Extinction Effect of Gold Nanocatalysts on Photocatalytic Activities under Plasmonic Excitation
}

\author{
Donghee Kim and Youngsoo Kim *D
}

check for

updates

Citation: Kim, D.; Kim, Y. Extinction Effect of Gold Nanocatalysts on Photocatalytic Activities under Plasmonic Excitation. Catalysts 2021, 11, 413. https://doi.org/10.3390/ catal11040413

Academic Editor: Vignesh Kumaravel

Received: 25 February 2021

Accepted: 21 March 2021

Published: 24 March 2021

Publisher's Note: MDPI stays neutral with regard to jurisdictional claims in published maps and institutional affiliations.

Copyright: (c) 2021 by the authors. Licensee MDPI, Basel, Switzerland. This article is an open access article distributed under the terms and conditions of the Creative Commons Attribution (CC BY) license (https:/ / creativecommons.org/licenses/by/ $4.0 /)$.
Department of Chemistry, Yeungnam University, Gyeongsan, Gyeongbuk 38541, Korea; heeya9635@naver.com * Correspondence: kimys6553@yu.ac.kr; Tel.: +82-53-810-2355

\begin{abstract}
Plasmonic nanoparticles (NPs), particularly Au NPs, are potential candidates for photocatalysts because of their unique optical properties. The size of Au NPs plays a crucial role in effective light absorption, which is an important factor in photocatalytic reactions. Although Au NP-based photocatalysts have garnered significant researched interest, the size effect of Au NPs on a photocatalytic reaction has not been sufficiently studied. We characterized the effect of size on the photocatalytic activity of Au NPs of different sizes. We found that the absorption cross-section of the Au NPs gradually increased as the size of the Au NPs increased. However, the reaction rate for each size of NP was inversely proportional to the absorption cross-section. Based on the simulation results, we found that larger Au NPs have a higher scattering factor than that of smaller Au NPs. Consequently, the scattering effect of Au NPs hinders effective light absorption, resulting in slower reaction kinetics. These findings can contribute to the rational design of high-efficiency plasmonic photocatalysts.
\end{abstract}

Keywords: Au nanoparticle; photocatalysts; surface plasmon resonance; electron transfer kinetics; seeded-growth method

\section{Introduction}

Plasmonic noble metal nanoparticles (NPs) have received significant attention because of their unique optical properties in the visible light region and their catalytic activity in the nanoscale regime [1-4]. These properties result from their localized surface plasmon resonance (LSPR) and are advantageous for their implementation as photocatalysts. Plasmonic metal NPs serve as highly reactive species, named as hot electrons and hot holes, which can be involved in a chemical transformation by interaction with visible light [5-8]. In earlier studies, hetero-structured nanomaterials, which are composed of metal and metal oxide NPs, were mainly used as visible-light-driven plasmonic photocatalysts. Heterostructured plasmonic photocatalysts drive important chemical transformations such as the dissociation of $\mathrm{H}_{2}$, ethane epoxidation, water splitting, and reduction of $\mathrm{CO}_{2}$ to hydrocarbons [5,9-12]. In such photocatalysts, the role of the plasmonic NPs is limited to a light-absorbing antennae, and the metal oxide NPs act as the reaction center by transferring electrons from metal NPs. Although the excited electron is generated only in metal NPs by light, the metal NP (mostly Au) itself is not considered as a potential plasmonic photocatalyst because the timescale of energy relaxation of photoexcited charge carriers is extremely small [13], and thus kinetically redundant in a chemical reaction. However, some studies on photochemical reactions with plasmonic Au photocatalysts have been recently reported. Kim et al. reported the harvesting of energetic charge carriers in Au NPs, the amount of activation energy that is reduced under plasmonic excitation, and the transference of excited electrons to the reaction substance through the insulating ligand in plasmonic $\mathrm{Au}$ photocatalysts [14-16]. Furthermore, multi-electron transfer $\mathrm{CO}_{2}$ reduction [17], the coupling of photo-reduced carbon species [18], and the chemical potential of light by plasmonic excitation [19], were fully investigated by Jain et al. Despite the growing interest in plasmonic Au catalysts, the size of the Au NPs was fixed at approximately $15 \mathrm{~nm}$ in the 
studies mentioned above, and the yield of photoconversion was low. According to the Mie theory, the wavelength and intensity of the surface plasmon resonance for plasmonic NPs strongly depend on the diverse factors affecting the electron density of the particle, such as size, shape, dielectric constant of the surrounding medium, chemical composition, and type of metal [20,21]. The LSPR of Au NPs appears mostly over $500 \mathrm{~nm}$ due to the charge carrier density. The intensity and absorbance at the LSPR of Au NPs increase as the particle size increases (assuming identical shape and surrounding medium). It is anticipated that by controlling the size of Au NPs, the absorption cross-section and wavelength can be tuned to effectively generate photoexcited electrons, leading to relatively higher photoreactivity. In this study, we prepared Au NPs of four different sizes using two synthetic strategies called the Turkevich method and the seeded-growth method, and we analyzed the photocatalytic activity that occurred via the plasmon-driven photoreduction of ferricyanide $\left(\mathrm{Fe}(\mathrm{CN})_{6}{ }^{3-}\right.$ ) to ferrocyanide $\left(\mathrm{Fe}(\mathrm{CN})_{6}{ }^{4-}\right)$. We examined the reaction rate of each reaction condition under $532 \mathrm{~nm}$ excitation for all sizes of Au NPs. As anticipated, the absorption cross-section of the Au NPs increased as the number of Au NPs increased. However, we found that the larger Au NPs exhibited a slower reaction rate than that of $15 \mathrm{~nm}$ Au NPs, which is the most common size. As the absorption efficacy of Au NPs increased with an increase in size, the efficacy of light scattering and the ratio of scattering to absorption also increased. By correlating the reaction kinetics and extinction spectra of Au NPs of varying sizes, we found that the scattering effect could hinder the effective number of photons absorbed by the Au NPs.

\section{Results and Discussion}

Spherical Au NPs with a size range of 15-35 nm were synthesized with two synthetic methods called the Turkevich method and the seeded-growth method by citrate reduction. $\mathrm{Au}$ NPs with a diameter of $15 \mathrm{~nm}$ were synthesized by the Turkevich method, which yields a narrow size distribution, whereas Au NPs with diameters of 20, 25, and $35 \mathrm{~nm}$ were prepared using the seeded-growth method. Au NPs with a diameter range of 20-35 nm were prepared using the method proposed by Puntes and co-workers [22] with some modifications (described in detail in the Methods section). A schematic illustration of the modified seeded-growth method is depicted in Supplementary Materials (Figure S1). In the citrate reduction method, the concentration ratio of citrate to $\mathrm{HAuCl}_{4}$ is a key parameter for controlling the size of the $\mathrm{Au}$ NPs. When the concentration ratio of citrate to $\mathrm{HAuCl}_{4}$ was less than 2, the NPs were prone to aggregate such that their sizes increased and their shapes became irregular. However, by increasing the ratio up to 3.5, the size of the NPs further decreased; a further increase in the ratio resulted in a decrease in the size of the Au NPs, followed by gradual saturation [23]. Figure 1 shows the morphological characterization of the four different sizes of Au NPs prepared using the two methods and respective size distribution histograms. The concentration ratio of citrate to $\mathrm{HAuCl}_{4}$ for the seed solution was determined to be 13 , which was decreased to 6.5 by adding $1 \mathrm{~mL}$ of $\mathrm{HAuCl}_{4}$ solution. The size of Au NPs in the resulting solution after growth for 30 min, called G1 colloids, was determined to be ca. $20 \mathrm{~nm}$ (Figure 1b,f). For the preparation of Au NPs with a size of $25 \mathrm{~nm}$, a set of dilutions and injections was required. The concentration ratio of citrate to $\mathrm{HAuCl}_{4}$ after dilution was determined to be 11, which is close to the ratio of the $\mathrm{Au}$ seed, and it was determined to be 5.5 after the addition of $1 \mathrm{~mL}$ of $\mathrm{HAuCl}_{4}$. After $30 \mathrm{~min}$ of growth, the size of the Au NPs in the resulting solution, called G2 colloids, was determined to be ca. $25 \mathrm{~nm}$ (Figure 1c,g). An additional cycle of dilution and injection was performed to obtain Au NPs with a size of ca. $35 \mathrm{~nm}$, called G3 colloids (Figure 1d,h). The ratios in the final set of solutions were determined to be 10 and 5 , respectively. The concentration ratio of citrate to $\mathrm{HAuCl}_{4}$ was maintained during three consecutive growth steps. The size of $\mathrm{Au}$ NPs prepared by the conventional Turkevich method was determined to be ca. $15 \mathrm{~nm}$ with a very narrow distribution (Figure 1a,e). The average size and size distribution (standard deviation) of the synthesized Au NPs were determined from at least 240 randomly selected NPs from random areas of the transmission electron microscopy (TEM) grid. Based on the 
TEM results, we confirmed that well-dispersed spherical Au NPs were synthesized and their standard deviation was mostly within $\pm 2 \mathrm{~nm}$, which is a very narrow distribution.
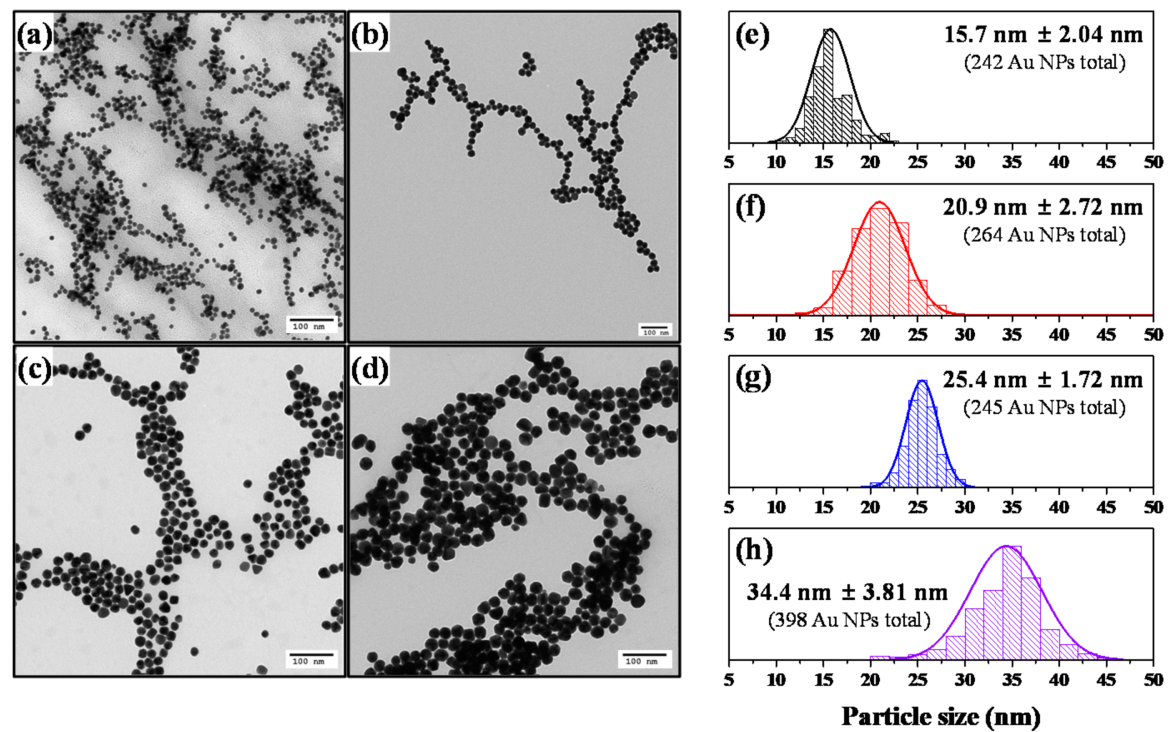

Figure 1. TEM images of Au NPs prepared by two methods with a size of (a) $15 \mathrm{~nm}$, (b) $20 \mathrm{~nm}$, (c) $25 \mathrm{~nm}$, and (d) $35 \mathrm{~nm}$. (e-h) Average size of Au NPs with a standard deviation. The value of average size for each NP was chosen from at least 240 NPs from random regions of the TEM grid.

The surface plasmon resonance features of the Au NPs prepared with four different sizes were examined by ultraviolet-visible (UV-Vis) absorption spectra, and we compared these spectra for each Au NP using the NANODDSCAT+ simulation tool. Figure 2a shows the extinction spectra of the as-prepared citrate-stabilized Au NPs, whose absorption bands for different sizes appeared at 520,523, 524, and $526 \mathrm{~nm}$. To further understand the optical properties of Au NPs with different sizes, we employed the open-source NANODDSCAT+ code from nanoHUB.org [24]. This simulation code, based on the discrete dipole approximation (DDA) method, was designed to study the LSPR spectrum and electric field of plasmonic NPs [25]. LSPR simulation results for Au NPs with different sizes are shown in Figure 2b. The simulated absorption spectra of the Au NPs appeared at 522, 523, 523, and $525 \mathrm{~nm}$. It should be noted that the dielectric constant of the capping agent, citrate, as a function of given wavelength was not considered in the simulation because the density of the capping agent in the vicinity of Au NPs with different sizes was uncertain. However, the peak of the LSPR band and the tendency in the peak shift in the simulated results are in good agreement with the experimental results. Figure $2 \mathrm{c}, \mathrm{d}$ show the peak absorbance and maximum LSPR wavelength of the as-synthesized Au NPs. As can be seen, the absorbance gradually increased, and the wavelength shifted to a longer wavelength as the size of the Au NPs increased. As anticipated, following the Mie theory, the surface plasmon resonance band of Au NPs shifted to a longer wavelength and its intensity increased with the increasing Au NP size. 

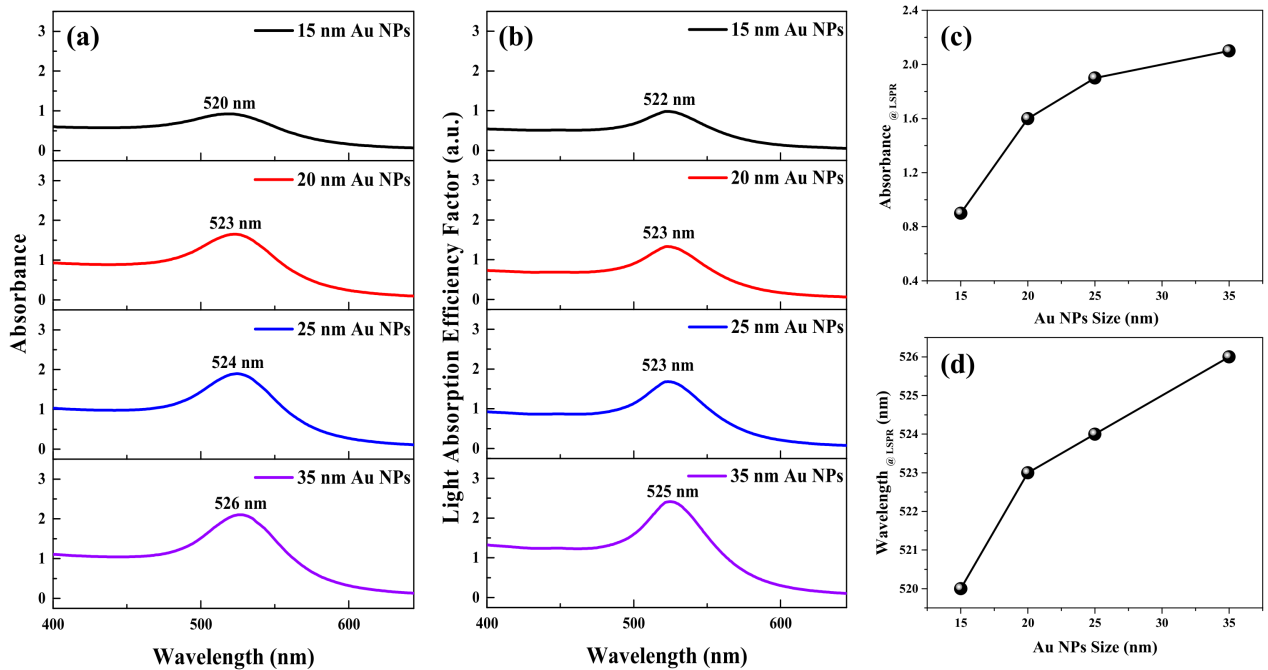

Figure 2. UV-Vis absorption spectra result by (a) experiment and (b) simulation by NANODDSCAT+. (c) Change in absorbance and (d) wavelength at LSPR as a function of particle size. The absorption band for different sizes of Au NPs from both experimental and simulation results is consistent, and the trajectory of absorbance and the wavelength at LSPR as a function of size follows the Mie theory.

The photocatalytic reaction involved the reduction of ferricyanide $\left(\mathrm{Fe}^{3+}\right)$ to ferrocyanide $\left(\mathrm{Fe}^{2+}\right)$ by photoexcited electron transfer from Au NPs. This reduction is a wellknown reaction catalyzed by transition metal nanostructures [26,27]. Recently, Kim et al. reported photocatalytic ferricyanide reduction by Au-catalyzed electron transfer, in which longer surviving photoexcited hot electrons in the conduction band of Au NPs participated in a ferricyanide reduction reaction by effectively capturing the holes remaining in the valence band [14-16]. For the charge neutralization of the reaction system, the holes in the valence band of Au NPs are involved in the oxidation reaction of ethanol, which is used as a hole scavenger [14-16]. For the photochemical reaction, the as-synthesized $\mathrm{Au}$ photocatalyst requires ligand exchange because citrate is prone to oxidation by complicated photochemistry [28]. Hence, we chose 11-mercaptoundecanoic acid (11-MUA), which has a good binding affinity to the Au surface and polarity to the reaction solvents. Lämmerhofer group has reported that qualified surface coverage of thiolated ligand (11-MUA) of Au NPs is estimated to be 5.7 molecules per $\mathrm{nm}^{2}$. The total number of molecules of 11-MUA that covered on the surface that used in our experiments could be simply estimated to be ca. 124 molecules for $15 \mathrm{~nm}$, ca. 220 molecules for $20 \mathrm{~nm}$, ca. 344 molecules for $25 \mathrm{~nm}$, and ca. 675 molecules for $35 \mathrm{~nm}$, respectively [29]. These are strongly bound onto the $\mathrm{Au}$ surface and are well-separated to minimize steric hindrance to each other.

We performed the photoreduction of ferricyanide according to the different sizes of $\mathrm{Au}$ NPs as a photocatalyst under a $532 \mathrm{~nm}$ continuous wave (CW) laser with a power of $700 \mathrm{~mW}$. Ethanol was used as the hole scavenger in the reaction mixture. We spectrophotometrically observed the reaction progress of this photocatalytic chemical reaction for each size of Au NP by monitoring the decrease in the absorption band of ferricyanide at $419 \mathrm{~nm}$ and increasing its absorbance at $240 \mathrm{~nm}$ (see Supplementary Materials Figure S2). The absorbance at $419 \mathrm{~nm}$ and $240 \mathrm{~nm}$ that correspond to $\mathrm{Fe}^{3+}$ and $\mathrm{Fe}^{2+}$ exceed the absorbance of 1 , which can make a huge error to determine concentration. Because the absorbance of the reaction mixture in the shorter wavelength was highly promoted by the contribution of Au NPs, the spectrum of the Au NPs should be eliminated from the reaction mixture. To minimize the error associated with measuring a concentration, we carried out the subtraction of the spectral contribution of Au NPs from the reaction mixture in a mathematical manner (see details in the method section). The absorbance at $419 \mathrm{~nm}$ and $240 \mathrm{~nm}$ after subtraction is able to consider more convincing to determine the concentration. The reaction rate as a function of the Au NPs size was determined by spectrophotometric observation (see Supplementary Materials, Figure S2). Figure 3 shows the increased con- 
centration as a function of irradiation time and as the size of Au NPs. Each concentration $\left(\mathrm{Fe}^{3+}\right.$ and $\left.\mathrm{Fe}^{2+}\right)$ was determined using the Beer-Lambert law at $419 \mathrm{~nm}$ and $240 \mathrm{~nm}$, and the molar absorption coefficient was used $1050 \mathrm{M}^{-1} \mathrm{~cm}^{-1}$ for Fe ${ }^{3+}$, and $6250 \mathrm{M}^{-1} \mathrm{~cm}^{-1}$ for $\mathrm{Fe}^{2+}$. The average concentration was obtained by taking the absolute values for each concentration (see details in the method section). The reaction rate was determined to be $1.9 \times 10^{-8} \mathrm{M} \cdot \mathrm{s}^{-1}$ for $15 \mathrm{~nm}, 1.2 \times 10^{-8} \mathrm{M} \cdot \mathrm{s}^{-1}$ for $20 \mathrm{~nm}, 0.6 \times 10^{-8} \mathrm{M} \cdot \mathrm{s}^{-1}$ for $25 \mathrm{~nm}$, and $0.5 \times 10^{-8} \mathrm{M} \cdot \mathrm{s}^{-1}$ for $35 \mathrm{~nm}$. According to the absorption spectra shown in Figure 2a, the absorption cross-section of Au NPs at the excitation wavelength gradually increases with increasing particle size. It was expected that a higher absorption cross-section would generate more electrons that can participate in the chemical reaction. However, the fastest reaction rate was observed in the $15 \mathrm{~nm} A u \mathrm{NPs}$, four times faster than that of the $35 \mathrm{~nm}$ $\mathrm{Au}$ NPs, which had the largest absorption cross-section in this experiment.
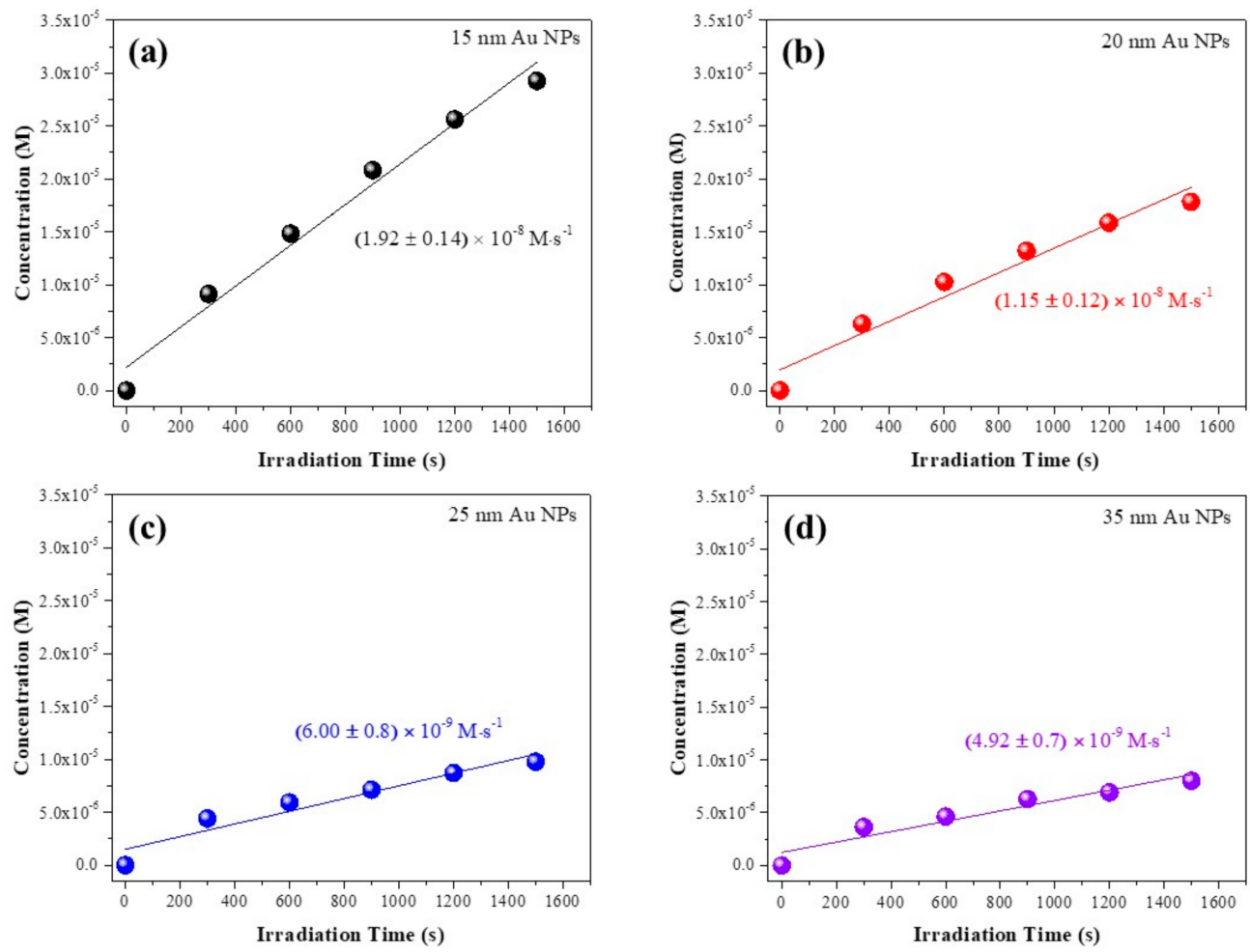

Figure 3. Au-catalyzed photochemical reaction under $532 \mathrm{~nm}$ light excitation with a power of $700 \mathrm{~mW}$. Concentration (M) as a function of irradiation time for (a) $15 \mathrm{~nm}$, (b) $20 \mathrm{~nm}$, (c) $25 \mathrm{~nm}$, and (d) $35 \mathrm{~nm}$ $\mathrm{Au}$ NPs. The values in each figure are reaction rates determined by linear fitting for each experiment.

We also examined the reaction kinetics and quantum efficiency as a function of the laser power for each $\mathrm{Au}$ NP. The laser power-dependent reaction rate was tabulated summarized in the Supplementary Materials (Table S1). The reduction reaction rarely occurred in the absence of light but in the presence of Au NPs. Even without light, ferricyanide molecules in the reaction mixture could readily diffuse to negatively charged Au NPs, and the surface electrons of Au NPs could be transferred to surface-adsorbed ferricyanide, leading to the generation of a reduced form of ferrocyanide. As seen in Figure $4 a$, the reaction rate in the absence of light gradually increased from larger particles to smaller particles. This could be attributed to the difference in the concentration of the Au NPs in the reaction mixture. The concentration of each size of Au NPs in the reaction mixture was determined using theoretically calculated extinction coefficient by Haiss, et al. [30] In the literature, the extinction coefficient at $450 \mathrm{~nm}$ for naked Au NPs was estimated to be $2.18 \times 10^{8} \mathrm{M}^{-1} \mathrm{~cm}^{-1}$ for $15 \mathrm{~nm}, 5.41 \times 10^{8} \mathrm{M}^{-1} \mathrm{~cm}^{-1}$ for $20 \mathrm{~nm}, 1.19 \times 10^{9} \mathrm{M}^{-1} \mathrm{~cm}^{-1}$ for $25 \mathrm{~nm}$, and $3.21 \times 10^{9} \mathrm{M}^{-1} \mathrm{~cm}^{-1}$ for $35 \mathrm{~nm}$, respectively. It should be noted that the dielectric constant of capping ligands does not take into account a calculation of the 
extinction coefficient of Au NPs in this literature. The effect of the dielectric constant makes an insignificant contribution to determine the extinction coefficient of the LSPR band of $\mathrm{Au}$ NPs. [31,32]. Taking this into the consideration, we calculated the concentration of $\mathrm{Au}$ NPs in the reaction mixture. The concentration of Au NPs was determined to be ca. $3.65 \times 10^{-9} \mathrm{M}$ for $15 \mathrm{~nm}$, ca. $1.29 \times 10^{-9} \mathrm{M}$ for $20 \mathrm{~nm}$, ca. $6.05 \times 10^{-10} \mathrm{M}$ for $25 \mathrm{~nm}$, and ca. $1.99 \times 10^{-10} \mathrm{M}$ for $35 \mathrm{~nm}$ of Au NPs. In the absence of light, the surface reaction between $\mathrm{Au}$ NPs and ferricyanide has occurred and the reaction rate was reflected by the effect of concentration of $\mathrm{Au}$ NPs in the reaction mixture. However, the reaction in the dark is much lower than under visible light illumination. To make an identical number of photons absorbed at the excitation wavelength, the absorbance of the reaction mixture for each $\mathrm{Au}$ $\mathrm{NP}$ size was set to ca. 1 at $532 \mathrm{~nm}$. As explained earlier, the absorbance as a function of the NP size is quite different. The absorption cross-section of Au NPs with a size of $15 \mathrm{~nm}$ was significantly lower than that of $35 \mathrm{~nm}$ Au NPs. Thus, the total number of NPs in each reaction mixture, including the specific size of the Au NPs, is inevitably different. This might be a reason to influence the reaction rate in the absence of light. In contrast, the total number of NPs in the reaction vessel was not a dominant factor in the presence of light. Under visible light excitation, the Au NPs generate photoexcited electrons, which are readily injected into ferricyanide, leading to the formation of ferrocyanide. Hence, photoexcited electron transfer is the key parameter for a chemical reaction in the presence of visible light. With increasing laser power, which leads to the increase in incident photons, the reaction rate increases. The trajectory of the reaction rate as a function of laser power for $15 \mathrm{~nm}$ and $20 \mathrm{~nm}$ Au NPs is quite similar to that of previously reported results [14]. However, the reaction rates for $25 \mathrm{~nm}$ and $35 \mathrm{~nm}$ Au NPs did not increase significantly with the increasing laser power. The difference in the reaction rate as a function of laser power increases with increasing laser power. This implies that either the light absorption process did not effectively occur or the photoexcited electrons could not be sufficiently transferred.

(a)

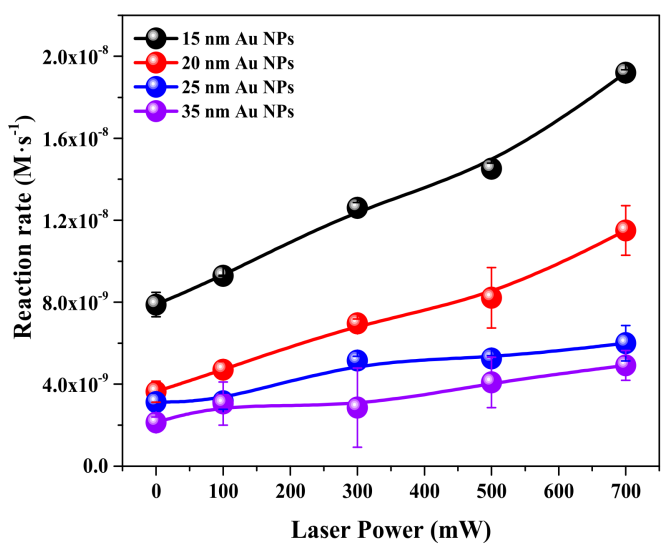

(b)

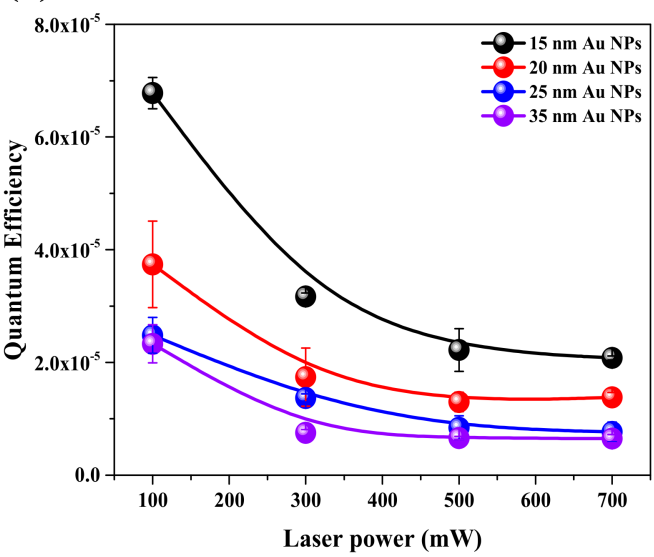

Figure 4. (a) Reaction rate and (b) quantum efficiency as a function of incident photon flux. The data points in each figure were obtained by average values obtained in at least three independent experiments under identical conditions. The error bars indicate the standard deviation taken from each identical experiment. The solid lines in the figure are visual guides.

The quantum efficiency of the photochemical reaction by electron transfer is defined as the efficiency of converting absorbed light into chemical transformation [33]. Typically, the quantum efficiency in photocatalytic reactions decreases exponentially with an increase in the number of incident photons. While charge separation and fast recombination events continuously occur under light excitation, the rate of electron transfer is governed by the diffusion of chemical substances in the reaction. Even if a large number of electrons are generated at a high power, most of these excited electrons quickly recombine and do not participate in the chemical reaction, resulting in a steep decrease in quantum efficiency. 
Figure $4 \mathrm{~b}$ shows the calculated quantum efficiency for each Au NP size as a function of laser power, and the quantum yield values are summarized in Table 1. At the lowest laser power $(100 \mathrm{~mW})$, the calculated quantum yield for $15 \mathrm{~nm}$ and $35 \mathrm{~nm}$ Au NPs was estimated to be ca. $6.78 \times 10^{-5}$ and ca. $2.33 \times 10^{-5}$, respectively. The quantum yield gradually decreases and then stabilizes, eventually saturating as the incident number of photons increases. These tendencies of quantum yield as a function of laser power were identically observed for all sizes of Au NPs. The quantum yield at the highest power $(700 \mathrm{~mW})$ for $15 \mathrm{~nm}$ and $35 \mathrm{~nm}$ Au NPs was estimated to be ca. $2.33 \times 10^{-5}$ and ca. $6.50 \times 10^{-6}$, respectively. Interestingly, the quantum yield of the smallest Au NPs used in this experiment was three times higher than that of the largest Au NPs under all irradiation conditions. These results indicate that $15 \mathrm{~nm}$ Au NPs can convert chemical substances more efficiently under the same amount of light absorbed.

Table 1. Summarized quantum efficiency as a function of laser power for Au NPs with varying sizes.

\begin{tabular}{ccccc}
\hline $\begin{array}{c}\text { Laser Power } \\
(\mathbf{m W})\end{array}$ & $\mathbf{1 5} \mathbf{~ n m}$ & \multicolumn{2}{c}{ Quantum Efficiency } \\
$\mathbf{2 0} \mathbf{~ n m}$ & $\mathbf{2 5} \mathbf{~ n m}$ & $\mathbf{3 5} \mathbf{~ n m}$ \\
\hline 100 & $6.78 \times 10^{-5}$ & $3.74 \times 10^{-5}$ & $2.48 \times 10^{-5}$ & $2.33 \times 10^{-5}$ \\
300 & $3.17 \times 10^{-5}$ & $1.74 \times 10^{-5}$ & $1.37 \times 10^{-5}$ & $7.50 \times 10^{-6}$ \\
500 & $2.22 \times 10^{-5}$ & $1.30 \times 10^{-5}$ & $8.43 \times 10^{-6}$ & $6.58 \times 10^{-6}$ \\
700 & $2.08 \times 10^{-5}$ & $1.38 \times 10^{-5}$ & $7.68 \times 10^{-6}$ & $6.50 \times 10^{-6}$ \\
\hline
\end{tabular}

In general, the total number of hot electrons generated by absorbing light in Au NPs is directly proportional to the number of photons absorbed, and the reaction rate is determined by the total number of electrons that can participate in a chemical reaction per second in electron transfer kinetics. Contrary to our expectation, the results showed that the highest reaction rate and quantum yield were observed for the $15 \mathrm{~nm}$ Au NPs, which have the smallest light absorption efficacy. Our experimental results imply that the efficacy of light absorption is not the dominant factor in determining the reaction kinetics of the photochemical reaction. Hence, from the perspective of photochemical reactions, it is necessary to consider both light absorption and light scattering. The NANODDSCAT+ tool can be used in simulations to provide both light-absorption efficiency and light-scattering efficiency with the given nanostructures (See Supplementary Materials, Figure S3). Figure 5a shows the simulated light scattering efficiency for each Au NP size. For $15 \mathrm{~nm}$ Au NPs, the scattering efficiency is close to zero, which means that the effect of light scattering is negligibly low. However, the scattering efficiency increases with increasing Au NP size and steeply increases when the Au NP size reaches $35 \mathrm{~nm}$. According to the Mie scattering theory, the cross-section of light scattered by small particles is given as follows $[34,35]$ :

$$
\sigma_{\mathrm{sc}}=\frac{24 \pi^{3} \mathrm{~V}^{2} \varepsilon_{\mathrm{m}}^{2}}{\lambda^{4}}\left[\frac{\left(\varepsilon_{1}-\varepsilon_{\mathrm{m}}\right)^{2}+\varepsilon_{2}^{2}}{\left(\varepsilon_{1}+2 \varepsilon_{\mathrm{m}}\right)^{2}+\varepsilon_{2}^{2}}\right]
$$

where $\mathrm{V}$ is the volume of the NP, $\varepsilon_{\mathrm{m}}$ is the dielectric function of the surrounding medium, $\lambda$ is the wavelength of the incident light, and $\varepsilon_{1}+\mathrm{i} \varepsilon_{2}$ is the complex dielectric function of the NPs [35]. The total volume of $35 \mathrm{~nm}$ Au NPs was estimated to be 22,449 $\mathrm{nm}^{3}$, which is twelve times larger than that of Au NPs with a diameter of $15 \mathrm{~nm}$. The scattering efficiency for all sizes of Au NPs employed was only reflected by the volume, rather than the dielectric function or excitation wavelength. From the results of Figures $2 a$ and $5 a$, we tentatively conclude that the Au NPs with expanded volume simultaneously absorb more light and scatter more light. Figure $5 \mathrm{~b}$ shows the ratio of light scattering to light absorption as a function of the NP diameter. For $15 \mathrm{~nm}$ Au NPs, the value of the ratio is close to zero. In the case of $15 \mathrm{~nm}$, the contribution of light scattering is negligible compared to the contribution of light absorption. Even if the absolute value of the ratio of light scattering to absorption for each size of Au NP is inadequate for the absorption value, the relative contribution of light scattering is predominant with the increase in the size of Au NPs. 
With regard to the reaction rate and relative scattering contribution as a function of the size of $\mathrm{Au} \mathrm{NPs,} \mathrm{it} \mathrm{is} \mathrm{considered} \mathrm{that} \mathrm{a} \mathrm{higher} \mathrm{contribution} \mathrm{of} \mathrm{light} \mathrm{scattering} \mathrm{results} \mathrm{in} \mathrm{a} \mathrm{slower}$ reaction rate. This suggests that light scattering hinders the effective number of photons absorbed by the Au NPs, leading to a reduction in the generation of photoexcited charge carriers.

(a)

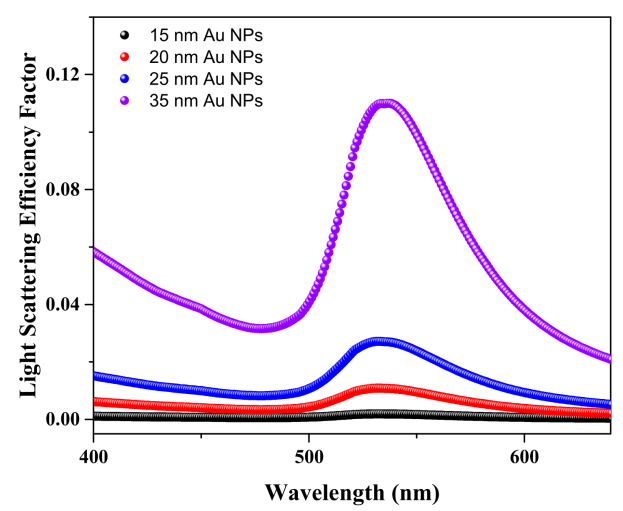

(b)

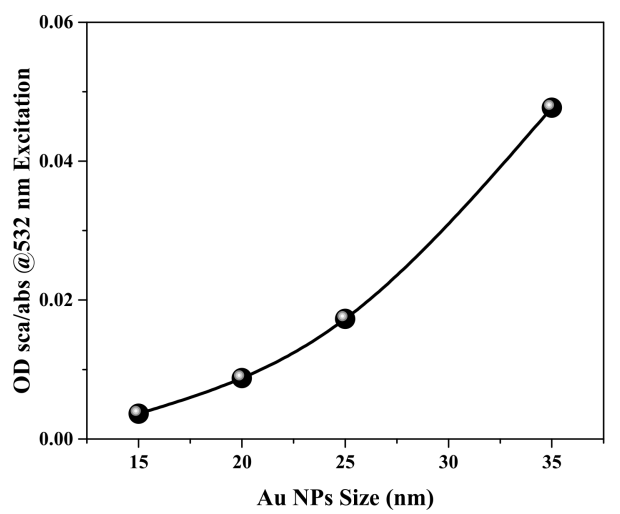

Figure 5. (a) Simulation plot for light scattering efficiency as a function of the size, obtained by NANODDSCAT+. (b) Ratio of scattering to absorption (ODsca/abs) at the plasmon excitation wavelength. The solid line in figure $(\mathbf{b})$ is a visual guide.

\section{Materials and Methods}

\subsection{Materials}

$\mathrm{Au}$ (III) chloride trihydrate $\left(\mathrm{HAuCl}_{4} \cdot 3 \mathrm{H}_{2} \mathrm{O}, \geq 49 \%\right.$ metal basis), 11-MUA (95\% purity), and potassium ferricyanide $\left(\mathrm{K}_{3} \mathrm{Fe}(\mathrm{CN})_{6}\right)$ were purchased from Sigma-Aldrich (St. Louis, MO, USA). Trisodium citrate dihydrate (99\% purity) was purchased from Alfa Aesar (Haverhill, MA, USA). All chemicals used were of analytical grade and were used as received without further purification. All water for the aqueous solution was purified using a water purification system (resistivity, $18.2 \mathrm{M} \Omega \cdot \mathrm{cm}$ at $25^{\circ} \mathrm{C}$, Daihan Scientific, Wonju, Korea).

\subsection{Synthetic Procedures of Au Nanoparticles}

\subsubsection{Synthesis of $15 \mathrm{~nm}$ Au Nanoparticles (Turkevich Method)}

Spherical Au NPs with a size of ca. $15 \mathrm{~nm}$ were synthesized by the Turkevich method. In a typical synthesis, $10 \mathrm{mg}$ of $\mathrm{HAuCl}_{4} \cdot 3 \mathrm{H}_{2} \mathrm{O}(0.025 \mathrm{mmol})$ was dissolved in $100 \mathrm{~mL}$ of deionized (DI) water in a $250 \mathrm{~nm}$ two-neck flask under vigorous stirring. In the initial stage, the solution was pale yellow. The solution was then heated until it started to boil. At this point, $0.6 \mathrm{~mL}$ of $0.17 \mathrm{M}$ of trisodium citrate solution was quickly injected. The color of the mixture solution changed from pale yellow to gray and then eventually wine-red within $10 \mathrm{~min}$. After the synthesis was completed, the flask was removed from the heating equipment, and the solution was allowed to naturally cool to room temperature.

\subsubsection{Synthesis of Au Nanoparticles with a Size of Up to $35 \mathrm{~nm}$ (Seeded-Growth Method)}

Au NPs with a size of up to $35 \mathrm{~nm}$ were prepared according to the seeded-growth method with some modifications [22]. For the preparation of Au seeds, $150 \mathrm{~mL}$ of an aqueous solution containing trisodium citrate $(2.2 \mathrm{mM})$ was prepared in a $250 \mathrm{~mL}$ two-neck flask equipped with a reflux condenser, and the solution was heated to the boiling point. After the solution started to boil, $1 \mathrm{~mL}$ of $\mathrm{HAuCl}_{4}(25 \mathrm{mM})$ was quickly injected while the solution was vigorously stirred. The solution was allowed to stand for $10 \mathrm{~min}$. At this time, the color of the solution changed from gray to pale pink. For the synthesis of Au NPs with diameters of 20,25, and $35 \mathrm{~nm}$, the reaction vessel containing Au seeds was allowed to cool naturally. When the temperature of the solution reached $90{ }^{\circ} \mathrm{C}, 1 \mathrm{~mL}$ of $\mathrm{HAuCl}_{4}$ solution 
$(25 \mathrm{mM})$ was injected. The solution was heated again and kept for $30 \mathrm{~min}$ at the boiling point to prepare $20 \mathrm{~nm}$ Au NPs (G1 colloids). After the first growth was completed, $55 \mathrm{~mL}$ of G1 colloids were extracted from the reaction vessel, and the leftover G1 solution in the reaction vessel was diluted by adding $53 \mathrm{~mL}$ of DI water and $2 \mathrm{~mL}$ of trisodium citrate $(60 \mathrm{mM})$. Diluted G1 colloids were used as seed particles for subsequent growth. The solution was heated to the boiling point again; another $1 \mathrm{~mL}$ of $\mathrm{HAuCl}_{4}$ solution $(25 \mathrm{mM})$ was injected, and the solution was kept for $30 \mathrm{~min}$ to achieve the growth of $25 \mathrm{~nm}$ Au NPs (G2 colloids). The largest ( $35 \mathrm{~nm}$ ) Au NPs were synthesized by repeating one more cycle of dilution and injection.

\subsubsection{Ligand Exchange of As-Synthesized Au Nanoparticles}

Thiolate-stabilized Au NPs were prepared for all synthesized Au NPs according to the following procedure. A $50 \mu \mathrm{L}$ solution of 11-MUA $(0.2 \mathrm{mM})$ ethanolic solution was mixed with $5 \mathrm{~mL}$ of citrate-stabilized Au NP colloid. Ultra-sonication was performed for $30 \mathrm{~min}$, and centrifugation was performed at 10,000 rpm for $15 \mathrm{~min}$. After centrifugation, the supernatant was carefully removed, and the precipitated Au NPs were re-dispersed in $1 \mathrm{~mL}$ of DI water and used for photocatalytic experiments.

\subsection{Characterization of Au Nanoparticles with Varying Sizes}

A TEM (H-7600, Hitachi, Tokyo, Japan) equipped with an AMT camera was used to characterize the size and morphology of Au NPs with varying sizes. The sizes of the four different $\mathrm{Au}$ NPs were determined using ImageJ, which is an open-source image manipulation tool. The optical properties of the synthesized Au colloids and the reaction mixture were measured using a Shimadzu UV-1800 spectrophotometer with a range of $200-900 \mathrm{~nm}$.

\subsection{Photocatalytic Reactions with Au Nanoparticles}

The reaction mixture was prepared as described elsewhere, with some modifications [14-16]. In this procedure, $1 \mathrm{~mL}$ of concentrated Au NP (absorbance ca. 3 at $532 \mathrm{~nm}$ ) colloid was mixed with $0.5 \mathrm{~mL}$ of ethanol and $1.47 \mathrm{~mL}$ of DI water, and $30 \mu \mathrm{L}$ of aqueous $50 \mathrm{mM}$ solution of potassium ferrycyanide $\left(\mathrm{K}_{3} \mathrm{Fe}(\mathrm{CN})_{6}\right)$ in a $3 \mathrm{~mL}$ quartz cuvette. The peak absorbance of the surface plasmon band of the reaction mixture was ca. 1 at the excitation wavelength for each NP size, and the initial concentration of $\mathrm{Fe}(\mathrm{CN})_{6}{ }^{3-}$ was $500 \mu \mathrm{M}$. The reaction mixture was purged for $15 \mathrm{~min}$ with Ar gas to ensure anaerobic conditions, and the deaerated reaction mixture was tightly sealed with a screw cap and $3 \mathrm{M}$ electrical tape to prevent air exposure. A solid-state $532 \mathrm{~nm}$ CW laser (CNI Laser, Changchun, China) was used as the monochromatic excitation source. The output power of the laser was measured using a power meter (ThorLabs PM100D) equipped with a thermal power sensor (ThorLabs S310C). The cuvette containing the reaction mixture was placed in a sample holder and irradiated with a laser beam while stirring. The change in absorbance of the reaction mixture by the photocatalytic reaction was recorded every $5 \mathrm{~min}$ of laser irradiation. The total reaction time was $25 \mathrm{~min}$. For experiments in the dark, the procedure was identical, except for the light irradiation. Additionally, control experiments were performed to determine the photoreactivity of $\mathrm{Fe}(\mathrm{CN})_{6}{ }^{3-}$ under $532 \mathrm{~nm}$ excitation. In these experiments, $2.47 \mathrm{~mL}$ of DI water was mixed with $0.5 \mathrm{~mL}$ of ethanol and $30 \mu \mathrm{L}$ of aqueous $50 \mathrm{mM} \mathrm{Fe}(\mathrm{CN})_{6}{ }^{3-}$, and all other experimental sequences were identical.

\subsection{Data Analysis (Molarity, Reaction Rate, and Quantum Efficiency)}

To determine the change in the concentration of the reaction mixture as a function of irradiation time, UV-Vis absorption spectra were recorded. The decrease in absorbance of $\mathrm{Fe}^{3+}\left(\mathrm{Fe}(\mathrm{CN})_{6}{ }^{3-}\right)$, the increase in absorbance of $\mathrm{Fe}^{2+}\left(\mathrm{Fe}(\mathrm{CN})_{6}{ }^{4-}\right)$, and the apparent isosbestic point were evidence of photoconversion. The photoconversion determination procedure was performed as described elsewhere. First, to determine the absolute con- 
centration of both $\mathrm{Fe}^{3+}$ and $\mathrm{Fe}^{2+}$, the contribution of $\mathrm{Au} \mathrm{NP}$ absorbance must be removed from the extinction spectrum of the reaction mixture as follows [14,15]:

$$
\text { Spectrum }_{\text {corr. }}=\text { Spectrum }_{\text {sample }}-\left(\text { Peak_Abs } \text { sample }_{\text {Peak_Abs }} / \text { Au NP }\right) \times \text { Spectrum }_{\text {AuNP }}
$$

Spectrum $_{\text {sample }}$ represents the absorption spectrum of the reaction mixture for the photocatalytic reaction, and Spectrum $\mathrm{Au}_{\mathrm{NP}}$ denotes the absorption spectrum of Au NP colloids, which is the same amount of Au NPs as that used in the reaction. The peak absorbance denotes the relative difference in absorbance between the peak absorbance at the Au LSPR and the absorbance at $800 \mathrm{~nm}$, where no absorbance was observed in both pure $\mathrm{Au}$ NPs and the reaction mixture. Next, as pure ferricyanide $\left(\mathrm{Fe}^{3+}\right)$ and ferrocyanide $\left(\mathrm{Fe}^{2+}\right)$ do not have any absorbance at $500 \mathrm{~nm}$, the absorbance at $500 \mathrm{~nm}$ after subtracting any trace absorbance from each spectrum must be zero. Following this correction, the change in absolute molarity as a function of reaction time, $\mathrm{t}$, was experimentally determined using the Beer-Lambert law. The change in the concentration of $\mathrm{Fe}^{3+}$ was calculated as

$$
\text { Concentration }(\mathrm{t})_{\mathrm{Fe}^{3+}}=-\frac{\left(\mathrm{A}_{419 \mathrm{~nm}}(\mathrm{t})-\mathrm{A}_{419 \mathrm{~nm}}(\mathrm{t}=\mathrm{o})\right)}{1050 \mathrm{M}^{-1} \mathrm{~cm}^{-1}}
$$

where the molar extinction coefficient of $\mathrm{Fe}^{3+}$ at $419 \mathrm{~nm}$ was $1050 \mathrm{M}^{-1} \mathrm{~cm}^{-1}$. Similar to the determination of $\mathrm{Fe}^{3+}$ concentration, the change in molarity of $\mathrm{Fe}^{2+}$ was determined as

$$
\text { Concentration }(t)_{\mathrm{Fe}^{2+}}=\frac{\left(\mathrm{A}_{240 \mathrm{~nm}}(\mathrm{t})-\mathrm{A}_{240 \mathrm{~nm}}(\mathrm{t}=\mathrm{o})\right)}{6250 \mathrm{M}^{-1} \mathrm{~cm}^{-1}}
$$

where the molar extinction coefficient of $\mathrm{Fe}^{2+}$ at $240 \mathrm{~nm}$ is $6250 \mathrm{M}^{-1} \mathrm{~cm}^{-1}$. The molar absorption coefficient of $\mathrm{Fe}^{3+}$ and $\mathrm{Fe}^{2+}$ was determined experimentally using Beer-Lambert law. The determined concentration from Equations (3) and (4) should ideally be the same, but systematic errors associated with spectrometry measurement result in mismatches on the calculated concentration of the two values. Thus, the average value of the two concentrations was used to determine the molarity. The determined concentration was plotted against irradiation time. The plot for each experiment was fitted to a straight line, and the slope corresponds to the reaction rate $(\mathrm{k})$ in $\mathrm{M} \mathrm{s}^{-1}$. The quantum efficiency (quantum yield) of the photocatalytic reaction was calculated as follows:

$$
\text { Quantum Efficiency }=\frac{N_{A} \times \text { Total conversion }(\text { in moles })}{\text { Number of photons incident } / \mathrm{s} \times\left(1-10^{-\mathrm{A}}\right) \times 1500 \mathrm{~s}}
$$

\subsection{NANODDSCAT+ Simulation}

The simulation of the LSRP spectra of Au NPs was performed using the DDA method. For this simulation, we employed the open-source NANODDSCAT+, which combines the discrete dipole scattering (DDSCAT) tool with the open-source 3D design tool "Blender" [25]. The target sizes of Au NPs, that is, 15, 20, 25, and $35 \mathrm{~nm}$, were generated with the design tool, and then "DDSCAT Convert" was used to discretize the 3D virtual dipole array as the input source for DDSCAT. From the virtual dipole array information, the absorption and scattering spectra for each Au NP size were simulated. This open-source simulation tool is available at nanoHUB.org [36-38].

\section{Conclusions}

We successfully synthesized Au NPs with diameters of 15, 20, 25, and $35 \mathrm{~nm}$ using the Turkevich method and the seeded-growth method. In addition, we characterized the effect of the size of Au NPs on a photochemical reaction under monochromatic light excitation. Our experimental and simulation results indicated that, as the particle size increased, the light absorption efficacy increased, and the influence of light scattering was enhanced. However, the reaction rate significantly decreased, while the ratio of scattering to absorption exponentially increased as the particle size increased. This implies that 
the light absorption on the Au NPs was strongly hindered by the light scattering effect, resulting in a decrease in the reaction rate. Thus, Au NPs with an appropriate ratio of light absorption and scattering can exhibit a better performance in plasmonic photocatalysts.

Supplementary Materials: The following are available online at https:/ / www.mdpi.com/2073-434 4/11/4/413/s1, Figure S1: Schematic illustration of the seeded-growth method, Figure S2: Spectroscopic monitoring of photocatalytic reduction of $\mathrm{Fe}^{3+}$ for each size of Au nanoparticles, Figure S3: Simulation of light-absorption and light-scattering spectra using NANODDSCAT+, Table S1: Summary of power-dependent reaction rate.

Author Contributions: D.K. conducted the experiments, performed data analysis, and performed the simulation. Y.K. conceived the research, designed the experiments, performed the simulation, performed the data analysis, and wrote the manuscript. All authors have read and agreed to the published version of the manuscript.

Funding: This work was supported by a grant from the National Research Foundation of Korea (NRF) funded by the Korean government (MSIT) (NRF-2019R1A2C1011692) and the 2018 Yeungnam University Research Grant.

Data Availability Statement: All data are shown in this articles and Supplementary Materials.

Acknowledgments: The authors thank the Core Research Support Center for Natural Products and Medical Materials (CRCNM) for technical support regarding the TEM measurements.

Conflicts of Interest: The authors declare no conflict of interest.

\section{References}

1. Kelly, K.L.; Coronado, E.; Zhao, L.L.; Schatz, G.C. The optical properties of metal nanoparticles: The influence of size, shape, and dielectric environment. J. Phys. Chem. B 2002, 107, 668-677. [CrossRef]

2. Link, S.; El-Sayed, M.A. Optical properties and ultrafast dynamics of metallic nanocrystals. Annu. Rev. Phys. Chem. 2003, 54, 331-366. [CrossRef] [PubMed]

3. Behl, M.; Jain, P.K. Catalytic activation of a solid oxide in electronic contact with gold nanoparticles. Angew. Chemie Int. Ed. 2015, 54, 992-997. [CrossRef] [PubMed]

4. Schuller, J.A.; Barnard, E.S.; Wenshan, C.; Jun, Y.C.; White, J.S.; Brongersma, M.L. Plasmonics for extreme light concentration and manipulation. Nat. Mater. 2010, 9, 193-204. [CrossRef]

5. Clavero, C. Plasmon-induced hot-electron generation at nanoparticle/metal-oxide interfaces for photovoltaic and photocatalytic devices. Nat. Photonics 2014, 8, 95-103. [CrossRef]

6. Linic, S.; Christopher, P.; Ingram, D.B. Plasmonic-metal nanostructures for efficient conversion of solar to chemical energy. Nat. Mater. 2011, 10, 911-921. [CrossRef]

7. Christopher, P.; Xin, H.; Marimuthu, A.; Linic, S. Singular characteristics and unique chemical bond activation mechanisms of photocatalytic reactions on plasmonic nanostructures. Nat. Mater. 2012, 11, 1044-1050. [CrossRef]

8. Lee, J.; Mubeen, S.; Ji, X.; Stucky, G.D.; Moskovits, M. Plasmonic photoanodes for solar water splitting with visible light. Nano Lett. 2012, 12, 5014-5019. [CrossRef]

9. Mubeen, S.; Lee, J.; Singh, N.; Krämer, S.; Stucky, G.D.; Moskovits, M. An autonomous photosynthetic device in which all charge carriers derive from surface plasmons. Nat. Nanotechnol. 2013, 8, 247-251. [CrossRef]

10. Hou, W.; Hung, W.H.; Pavaskar, P.; Goeppert, A.; Aykol, M.; Cronin, S.B. Photocatalytic Conversion of $\mathrm{CO}_{2}$ to Hydrocarbon fuels via plasmon-eenhanced absorption and metallic interband transitions. ACS Catal. 2011, 1, 929-936. [CrossRef]

11. Watanabe, K.; Menzel, D.; Nilius, N.; Freund, H.-J. Photochemistry on metal nanoparticles. Chem. Rev. 2006, 106, 4301-4320. [CrossRef]

12. Mukherjee, S.; Zhou, L.; Goodman, A.M.; Large, N.; Ayala-Orozco, C.; Zhang, Y.; Nordlander, P.; Hala, N. Hot-Electron-Induced Dissociation of $\mathrm{H}_{2}$ on gold nanoparticles supported on $\mathrm{SiO}_{2}$. J. Am. Chem. Soc. 2014, 136, 64-67. [CrossRef]

13. Bigot, J.Y.; Halté, V.; Merle, J.-C.; Daunois, A. Electron dynamics in metallic nanoparticles. Chem. Phys. 2000, 251, 181-203. [CrossRef]

14. Kim, Y.; Smith, J.G.; Jain, P.K. Harvesting multiple electron-hole pairs generated through plasmonic excitation of Au nanoparticles. Nat. Chem. 2018, 10, 763-769. [CrossRef]

15. Kim, Y.; Dumett Torres, D.; Jain, P.K. Activation energies of plasmonic catalysts. Nano Lett. 2015, 6, 3399-3407. [CrossRef] [PubMed]

16. Kim, Y.; Wilson, A.J.; Jain, P.K. The nature of plasmonically assisted hot-electron transfer in a donor-bridge-acceptor complex. ACS Catal. 2017, 7, 4360-4365. [CrossRef]

17. Yu, S.J.; Wilson, A.; Heo, J.K.; Jain, P. Plasmonic control of multi-electron transfer and C-C coupling in visible-light-driven $\mathrm{CO}_{2}$ reduction on Au nanoparticles. Nano Lett. 2018, 18, 2189-2194. [CrossRef] [PubMed] 
18. Yu, S.; Jain, P.K. Plasmonic photosynthesis of C 1-C 3 hydrocarbons from carbon dioxide assisted by an ionic liquid. Nat. Commun. 2019, 10, 1-7. [CrossRef] [PubMed]

19. Yu, S.; Jain, P.K. The chemical potential of plasmonic excitations. Angew. Chem. Int. Ed. 2020, 59, 2085-2088. [CrossRef]

20. Huang, X.; El-Sayed, M.A. Gold nanoparticles: Optical properties and implementations in cancer diagnosis and photothermal therapy. J. Adv. Res. 2010, 1, 13-28. [CrossRef]

21. Jain, P.K.; Huang, X.; El-Sayed, I.H.; El-Sayed, M.A. Noble metals on the nanoscale: Optical and photothermal properties and some applications in imaging, sensing, biology, and medicine. Acc. Chem. Res. 2008, 41, 1578-1586. [CrossRef]

22. Bastús, N.G.; Comenge, J.; Puntes, V. Kinetically controlled seeded growth synthesis of citrate-stabilized gold nanoparticles of up to $200 \mathrm{~nm}$ : Size Focusing versus Ostwald Ripening. Langmuir 2011, 27, 11098-11105. [CrossRef]

23. Ji, X.; Song, X.; Li, J.; Bai, Y.; Yang, W.; Peng, X. Size control of gold nanocrystals in citrate reduction: The third role of citrate. J. Am. Chem. Soc. 2007, 129, 13939-13948. [CrossRef]

24. Fang, A.; White, S.L.; Masitas, R.A.; Zamborini, F.P.; Jain, P.K. One-to-one correlation between structure and optical response in a heterogeneous distribution of plasmonic constructs. J. Phys. Chem. C 2015, 119, 24086-24094. [CrossRef]

25. Fang, A.; White, S.; Jain, P.K.; Zamborini, F.P. Regioselective plasmonic coupling in metamolecular analogs of benzene derivatives. Nano Lett. 2014, 15, 542-548. [CrossRef] [PubMed]

26. Carregal-Romero, S.; Pérez-Juste, J.; Hervés, P.; Liz-Marzán, L.M.; Mulvaney, P. Colloidal gold-catalyzed reduction of ferrocyanate (III) by borohydride ions: A model system for redox catalysis. Langmuir 2009, 26, 1271-1277. [CrossRef]

27. Hervés, P.; Pérez-Lorenzo, M.; Liz-Marzán, L.M.; Dzubiella, J.; Lu, Y.; Ballauff, M. Catalysis by metallic nanoparticles in aqueous solution: Model reactions. Chem. Soc. Rev. 2012, 41, 5577-5587. [CrossRef] [PubMed]

28. Wu, X.; Thrall, E.S.; Liu, H.; Steigerwald, M.; Brus, L. Plasmon induced photovoltage and charge separation in citrate-stabilized gold nanoparticles. J. Phys. Chem. C 2010, 114, 12896-12899. [CrossRef]

29. Hinterwirth, H.; Kappel, S.; Waitz, T.; Prohaska, T.; Lindner, W.; Lämmerhofer, M. Quantifying thiol ligand density of selfassembled monolayers on gold nanoparticles by inductively coupled plasma-mass spectrometry. ACS Nano 2013, 7, 1129-1136. [CrossRef] [PubMed]

30. Haiss, W.; Thanh, N.T.K.; Aveyard, J.; Fernig, D.G. Determination of size and concentration of gold nanoparticles from UV-Vis spectra. Anal. Chem. 2007, 79, 4215-4221. [CrossRef]

31. Liu, X.; Atwater, M.; Wang, J.; Huo, Q. Extinction coefficient of gold nanoparticles with different sizes and different capping ligands. Colloids Surf. B Biointerfaces 2007, 58, 3-7. [CrossRef] [PubMed]

32. Oldenburg, S.J.; Averitt, R.D.; Halas, N.J. Nanoengineering of optical resonances. Chem. Phys. Lett. 1998, 288, 243-247. [CrossRef]

33. Marquez, D.M.; Sánchez, C.G. Quantum efficiency of the photo-induced electronic transfer in dye-TiO 2 complex. Phys. Chem. Chem. Phys. 2018, 20, 26280. [CrossRef] [PubMed]

34. Jain, P.K.; Lee, K.S.; El-Sayed, I.H.; El-Sayed, M.A. Calculated absorption and scattering properties of gold nanoparticles of different size, shape, and composition: Applications in biological imaging and biomedicine. J. Phys. Chem. B 2006, 110, 7238-7248. [CrossRef]

35. Anderson, L.J.E.; Mayer, K.M.; Fraleigh, R.D.; Yang, Y.; Lee, S.; Hafner, J.H. Quantitative measurements of individual gold nanoparticle scattering cross sections. J. Phys. Chem. C 2010, 114, 11127-11132. [CrossRef]

36. Flatau, P.J.; Draine, B.T. Fast near field calculations in the discrete dipole approximation for regular rectilinear grids. Opt. Express 2012, 20, 1247. [CrossRef] [PubMed]

37. Draine, B.T.; Flatau, P.J. User Guide to the Discrete Dipole Approximation Code DDSCAT 7.2. arXiv 2012, arXiv:1202.3424.

38. Smith, J.; Faucheaux, J.; White, S.; Sobh, A.N.; Feser, J.; Jain, P.K.; Sobh, N. nanoDDSCAT. 2014. Available online: https: //nanohub.org/resources/dda (accessed on 2 December 2020). [CrossRef] 